\title{
A Comprehensive Model of the Response of Silicon Photomultipliers
}

\author{
Herman T. van Dam, Stefan Seifert, Ruud Vinke, Peter Dendooven, Herbert Löhner, Freek J. Beekman, and \\ Dennis R. Schaart
}

\begin{abstract}
The response of a silicon photomultiplier (SiPM) to optical signals is inherently nonproportional due to saturation, afterpulsing, and crosstalk. Existing models of the SiPM response do not account for all of these effects, and therefore, these models are not sufficiently accurate for many applications. In this work, a comprehensive model of the SiPM response is developed that is generally applicable to exponentially decaying light pulses and that can be simplified in the case of very short (e.g., laser) light pulses. The model accounts for the total number and the temporal distribution of the incident photons as well as for the relevant SiPM parameters, viz. the recovery time, afterpulsing, crosstalk, and their cross correlations. The model is shown to correspond well with measurements on a SiPM-based scintillation detector. Furthermore, it is shown to be in agreement with several cases for which the SiPM response is known a priori. Having thus validated the model, its use is demonstrated by predicting the response of the Hamamatsu multipixel photon counter (MPPC) S10362-33-050C SiPM to several different scintillators.
\end{abstract}

Index Terms-Afterpulsing, crosstalk, multipixel photon counter (MPPC), nonproportionality, recovery, response model, scintillation detector, silicon photomultiplier, SiPM.

\section{INTRODUCTION}

$\mathbf{T}$ HE silicon photomultiplier (SiPM) - also referred to as, e.g., multipixel Geiger-mode avalanche photodiode (GM-APD or G-APD), multipixel photon counter (MPPC), solid-state photomultiplier (SSPM), single-photon avalanche diode (SPAD) array, or pixelated Geiger-mode avalanche photon detector (PPD) - is a relatively new type of low-level light sensor [1]-[7]. Since it is fast, has a high gain, is compact, and is potentially cost-effective, it is increasingly being applied in many areas, such as high-energy physics, medical imaging, astronomy, quantum optics, low-level (laser) light detection, photon counting, fluorescence spectroscopy, and biomolecular imaging [8]-[24].

Manuscript received January 15, 2010; revised April 09, 2010 and June 02, 2010; accepted June 08, 2010. Date of current version August 18, 2010. This work was supported by SenterNovem Grant IS055019.

H. T. van Dam, S. Seifert, and D. R. Schaart are with Delft University of Technology, Delft 2629 JB, The Netherlands (e-mail: d.r.schaart@tudelft.nl).

R. Vinke, P. Dendooven, and H. Löhner are with the Kernfysisch Versneller Instituut (KVI), Groningen 9747 AA, The Netherlands.

F. J. Beekman is with Delft University of Technology, Delft 2629 JB, The Netherlands, and also with University Medical Centre Utrecht, Utrecht 3584 CG, The Netherlands.

Color versions of one or more of the figures in this paper are available online at http://ieeexplore.ieee.org.

Digital Object Identifier 10.1109/TNS.2010.2053048
In contrast to many other photosensors, the performance of any SiPM-based detector may be affected significantly by the inherent nonproportionality of the SiPM response. Saturation, afterpulsing, and crosstalk are the main contributors in this, as they exhibit a nonlinear relationship to the number of incident photons detected in a given time interval. Because of these effects, it is not trivial to relate the measured, electronic signal to the corresponding optical signal, and vice versa. Nevertheless, for most applications it is desirable to understand and quantify the SiPM response, e.g., in order to properly design a detector for a given application, perform corrections on measurements or on energy spectra, calibrate a SiPM detector for low-level light measurements, predict detector performance, and/or determine difficult-to-measure SiPM parameters.

A commonly used simple exponential model of the SiPM response is only valid for instantaneous light pulses and assumes zero afterpulsing and crosstalk [25]. Models that account for the recovery of SiPM microcells have also been proposed, describing the SiPM output amplitude either for a sequence of instantaneous light pulses [26] or for light pulses of finite duration with a constant photon flux [27]. However, in many applications, these simple models are not sufficiently accurate, for example, because the influences of afterpulsing and crosstalk are significant, the duration of the light pulse is not negligible compared to the SiPM recovery time, and/or the photon flux is not constant. Also, the latter model is based on functions for which no physical justification is given [27].

The goal of the present work is to develop a comprehensive model of the SiPM response that is generally applicable to exponentially decaying light pulses. It accounts for the total number as well as the temporal distribution of the incident photons, and for the relevant SiPM parameters, viz. the SiPM recovery time, afterpulsing, crosstalk, and the cross correlations between these parameters. It will be shown that the model can be simplified in the case of short (e.g., laser) light pulses. The model is validated by comparison to measurements on a SiPM-based scintillation detector. Additionally, it is shown that the model agrees with several cases for which the response is known a priori. Having thus validated the model, its use is demonstrated by predicting the response of a Hamamatsu MPPC S10362-33-050C SiPM coupled to various scintillators.

\section{SiLICON PhOTOMULTIPLIERS}

SiPMs typically comprise between $\sim 10^{2}$ and $\sim 10^{5}$ parallelconnected GM-APDs, each reverse-biased at a voltage $V_{\text {bias }}$ and quenched by a resistor $R_{\mathrm{q}}$ [28], [29]. The SiPM output current can be converted into a voltage using a shunt resistor $R_{\mathrm{S}}$ and 


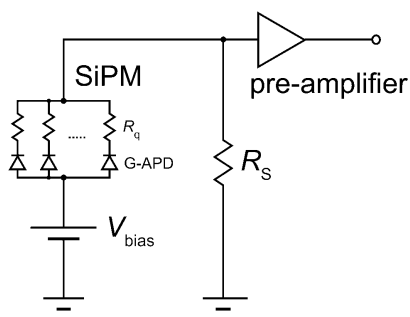

Fig. 1. Schematic overview of a SiPM and its readout circuit. The SiPM comprises $10^{2}-10^{5}$ GM-APDs, each quenched by a quenching resistor $R_{\mathrm{q}}$ and reverse-biased at $V_{\text {bias }}$. The output current of the SiPM is converted into a voltage by the shunt resistor $R_{\mathrm{S}}$ and amplified by a voltage preamplifier.

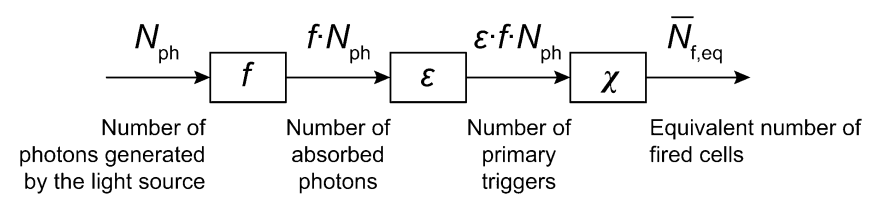

Fig. 2. Schematic overview of the light detection by a SiPM. As defined in Section II, $f$ denotes the photon collection efficiency, $\varepsilon$ the internal microcell $\mathrm{QE}$, and $f \cdot \varepsilon$ the detector PDE. The factor $\chi$ is the SiPM response discussed in Section III that accounts for the nonlinear relationship between the number of primary triggers and the measured output charge expressed in terms of the equivalent number of single fired cells. The measured output charge expressed in electrons can be obtained by multiplying $\bar{N}_{\mathrm{f}, \mathrm{eq}}$ with the SiPM gain $M$. It should be noted that for a proportional sensor such as a PMT or APD, the factor $\chi$ can be omitted, and $\varepsilon \cdot f \cdot N_{\mathrm{ph}}$ denotes the number of photoelectrons or electron-hole pairs, respectively.

amplified with a (high input impedance) voltage preamplifier as indicated in Fig. 1.

When a photon is absorbed in one of the GM-APDs, henceforth referred to as (micro)cells, an electron-hole pair may be created and, subsequently, a discharge may be triggered (i.e., the cell fires). These two processes of creating an electron-hole pair and triggering a discharge have a joint probability $\varepsilon(\lambda) \leq 1$, which we define as the internal quantum efficiency (QE) of a single microcell for incident photons with wavelength $\lambda$. For a light pulse consisting of photons with different wavelengths, the effective internal quantum efficiency $\varepsilon$ can be defined as the weighted average of $\varepsilon(\lambda)$. It should be noted that our definition of $\varepsilon$ is consistent with the one generally used for photosensors, i.e., the fraction of absorbed photons that contribute to the measured photocurrent. The internal microcell $\mathrm{QE}$ is to be distinguished from the external one, which includes the wavelengthand angle-dependent absorption probability for incident photons. Additionally, the SiPM photon detection efficiency (PDE), i.e., the product of the external microcell $\mathrm{QE}$ and the fill factor of the active area, is to be discerned from the so-called detector PDE of, e.g., a SiPM-based scintillation detector, which we define as the product of the internal microcell $\mathrm{QE} \varepsilon$ and the photon collection efficiency $f$ (i.e., the fraction of emitted photons that are absorbed in one or more microcells). The detector PDE thus includes effects related to the detector optical geometry, the use of absorbers, and/or reflectors, etc. This is illustrated schematically in the left part of Fig. 2.

The direct triggering of a discharge by a photon originating from the light source (e.g., a scintillator or laser) will be called a primary trigger in the remainder of this document. Other trigger mechanisms, such as afterpulsing, crosstalk, and dark counts, will be discussed in the next chapter. It is pointed out that for two simultaneous triggers in the same cell, the resulting output charge still corresponds to one fired cell. After a discharge the cell recovers and new photons can be detected. If a discharge is triggered while the cell is still recharging, the output charge corresponding to the second photon will be reduced. These effects are commonly referred to as saturation of the SiPM.

\section{A. SiPM Gain and Gain Nonproportionality}

A discharge in a microcell results in a current signal at the output of the SiPM. The gain $M$, defined as the expectation value of the number of output charge carriers due to a single fired cell, can be estimated as

$$
M=\left(C_{\mathrm{D}}+C_{\mathrm{q}}\right) V_{\mathrm{ob}} / e
$$

where $e$ is the elementary charge, $C_{\mathrm{D}}$ is the microcell capacitance, $C_{\mathrm{q}}$ is the parasitic capacitance of the quench resistor, and $V_{\mathrm{ob}}$ is the voltage-over-breakdown, defined as $V_{\mathrm{ob}}=V_{\text {bias }}-$ $V_{\mathrm{br}}$, where $V_{\mathrm{br}}$ is the breakdown voltage of the GM-APD [30].

For the remainder of this work, it is important to notice that the SiPM gain may not be constant during a light pulse with a duration of the same order of magnitude as the duration of the SiPM output pulse. This effect occurs if there is any resistance in series with the SiPM. In the readout scheme used in this work (see Fig. 1), the dominating resistance is $R_{\mathrm{S}}$. In other cases, this might be, for example, the input resistance of a charge-sensitive preamplifier (CSP) and/or the resistance of filter circuitry at the power supply lines.

If one or more photons have created a discharge, the signal current will cause a voltage drop over $R_{\mathrm{S}}$. This, in return, causes $V_{\mathrm{ob}}$ to be reduced by the same absolute value. Depending on the number of fired cells and the value of $R_{\mathrm{S}}$, this may lead to a significant reduction of $V_{\mathrm{ob}}$. The gain, being proportional to $V_{\mathrm{ob}}$, will be reduced by the same fraction. This effect is especially significant around the time when the voltage over $R_{\mathrm{S}}$ peaks. An example of such gain reduction is schematically shown in Fig. 3 as a function of time.

Experiments in which very short light (e.g., laser) pulses are used are not affected by this gain nonlinearity since in those cases the gain has not yet changed significantly at the time of arrival of any of the photons. As illustrated in Fig. 3, however, this effect may lead to a significant reduction of the average gain for (e.g., scintillation) light pulses with a duration of the same order of magnitude as the duration of the SiPM output pulse, depending on $R_{\mathrm{S}}, V_{\mathrm{ob}}$, and how many cells were fired at which times prior to the firing of a given cell.

It should be noted that, for fast pulses, the SiPM gain may show another type of electronic nonproportionality, which is typically in the order of a few percent and finds its origin in the variation of the time needed to quench the discharge as a function of the number of coincidently fired cells [29].

\section{SIPM RESPONSE}

Suppose a SiPM is illuminated by a light source generating a pulse of $N_{\mathrm{ph}}$ photons. As defined in Section II, the photon collection efficiency $f$ equals the fraction of these photons that are absorbed in one or more SiPM microcells, giving rise to a total expected number of $\bar{N}_{\text {abs }}=f N_{\mathrm{ph}}$ absorbed photons. 


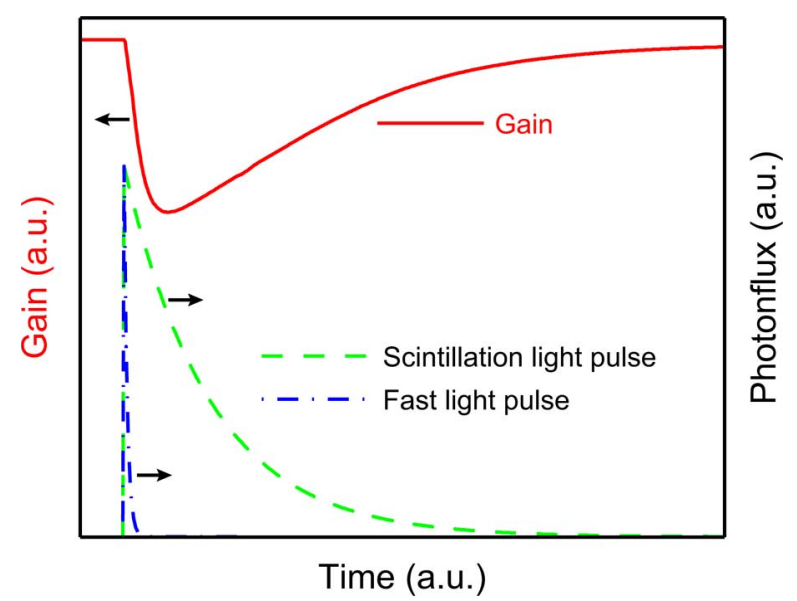

Fig. 3. Schematic representation of the SiPM gain nonproportionality as a function of time, together with a scintillation pulse and a fast light pulse, illustrating that part of the primary triggers may have a lower gain if the duration of the light pulse is of the same order of magnitude as the duration of the SiPM output pulse.

The corresponding expectation value of the number of primary triggers then equals $\varepsilon \bar{N}_{\mathrm{abs}}=\varepsilon f N_{\mathrm{ph}}$, where $\varepsilon \cdot f$ is the detector PDE discussed in Section II. We now define the response $\chi$ of a SiPM as the ratio between the expectation value of the total output charge $\bar{N}_{\text {f,eq }}$ and the expectation value of the number of primary triggers

$$
\chi \equiv \frac{\bar{N}_{\mathrm{f}, \mathrm{eq}}}{\varepsilon \bar{N}_{\mathrm{abs}}}=\frac{\bar{N}_{\mathrm{f}, \mathrm{eq}}}{\varepsilon f N_{\mathrm{ph}}}
$$

where $\bar{N}_{\text {f,eq }}$ is expressed in terms of the equivalent number of fired cells, defined as the ratio of the total signal charge and the charge due to a single fired microcell. (In this work, expectation values are denoted with a bar on top of the variable.) Fig. 2 schematically illustrates how $\chi$, together with $\varepsilon$ and $f$, determines the measured SiPM output charge $\bar{N}_{\mathrm{f}, \text { eq }}$ as a function of $N_{\text {ph }}$. It should be noted that the total SiPM output charge in terms of the number of electrons can be obtained by multiplying $\bar{N}_{\text {f,eq }}$ with the SiPM gain $M$ defined in (1).

Since $\bar{N}_{\text {f,eq }}$ depends nonlinearly on $\varepsilon, f, N_{\mathrm{ph}}$, afterpulsing, crosstalk, and saturation, the SiPM response $\chi$ is not constant. Specifically, if $\varepsilon \cdot f \cdot N_{\mathrm{ph}}$ is not small compared to the number of microcells $N_{\text {cells }}$, the response $\chi$ may be smaller than 1 due to saturation. Conversely, for $\varepsilon \cdot f \cdot N_{\text {ph }} \ll N_{\text {cells }}$ the response $\chi$ might be larger than 1 due to afterpulsing and crosstalk. It should be noted that $\chi$ is only defined for $\varepsilon \cdot f \cdot N_{\mathrm{ph}} \neq 0$.

\section{A. Lower Limit of SiPM Response}

A simple model of the effect of saturation on the response of a SiPM has been presented, e.g., by Stoykov et al. [25]. This model is applicable only to instantaneous light pulses and furthermore assumes that crosstalk, afterpulsing, and dark counts are absent. Under these conditions, the lower limit of the SiPM response $\chi_{\text {lower }}$ is reached, which can be written as

$$
\chi_{\text {lower }}=\frac{N_{\text {cells }}}{\varepsilon f N_{\mathrm{ph}}}\left(1-e^{-\frac{\varepsilon f N_{\mathrm{ph}}}{N_{\mathrm{cells}}}}\right) .
$$

\section{B. Factors Affecting the SiPM Response}

In case the light pulse is not instantaneous but has a finite duration, such as a scintillation light pulse, it is possible that cells are fired again after they have (partially) recovered from a previous discharge. In this case, still neglecting afterpulsing, crosstalk, and dark counts, $\chi_{\text {lower }}<\chi<1$.

A model for $\chi$ that accounts for the time profile of a scintillation light pulse, the SiPM recovery time, afterpulsing, crosstalk, dark counts, and their cross correlation requires an adequate description of these processes, taking into account their temporal characteristics. Here, it should be noted that many SiPM parameters are dependent on the voltage-over-breakdown $V_{\text {ob }}$ as well as on the temperature. Therefore, the response should be calculated for each value of $V_{\mathrm{ob}}$ and for each temperature separately.

1) Time Profile of a Scintillation Light Pulse: In a scintillator, optical photons are created upon absorption of $\gamma$-ray or X-ray radiation. In this work, it is assumed that the temporal distribution of this scintillation light pulse can be described adequately by a single exponential decay with time constant $\tau_{\mathrm{d}}$. It should be noted that the SiPM response model can relatively easily be adapted with a weighted sum of exponentials when necessary. The single-photon emission probability distribution $p_{\mathrm{sc}}(t)$ of a scintillation light pulse starting at $t_{0}=0$ is thus modeled as

$$
p_{\mathrm{sc}}(t)= \begin{cases}\frac{1}{\tau_{\mathrm{d}}} e^{-\frac{t}{\tau_{\mathrm{d}}}}, & t \geq 0 \\ 0, & t<0 .\end{cases}
$$

It is assumed that the traveling time spread of the optical photons in the scintillator is negligible, so the probability distribution of the photons incident on the light sensor and, therefore, of the primary triggers, can be described with (4) as well.

2) SiPM Recovery Time: Suppose a microcell is triggered at $t=t_{1}$ by an absorbed photon. The discharge of the microcell then causes a current $I_{\mathrm{q}}$ to flow through the quench resistor. An instantaneous discharge process is assumed since the discharging of $C_{\mathrm{D}}$ is at least an order of magnitude faster than the recharging. Then, the normalized current pulse $I_{\mathrm{q}, \text { norm }}$ through the quench resistor in response to a single discharge starting at $t=t_{1}$ can be described as

$$
I_{\mathrm{q}, \text { norm }}(t)= \begin{cases}\frac{1}{\tau_{\text {rec }}} e^{-\frac{t-t_{1}}{\tau_{\text {rec }}},} & t \geq t_{1} \\ 0, & t<t_{1}\end{cases}
$$

where $\tau_{\text {rec }}$ is the SiPM recovery time given by [31]

$$
\tau_{\text {rec }}=\left(C_{\mathrm{D}}+C_{\mathrm{q}}\right) R_{\mathrm{q}} \text {. }
$$

Suppose the microcell is fired again by a second absorbed photon at some time $t=t_{2}$ while it is still recharging. The current pulse then continues as of $t=t_{2}$ as a new exponential decay according to (5), but with $t_{1}$ replaced by $t_{2}$. This is illustrated in Fig. 4.

More generally, when a cell is fired $k_{\max }$ times and the time differences between triggers $k$ and $k-1$ are $\Delta t_{\mathrm{k}, \mathrm{k}-1}$, then the total output charge from a single microcell $N_{\mathrm{f}, \mathrm{eq}, 1}$, expressed in the equivalent number of fired cells, is

$$
N_{\mathrm{f}, \mathrm{eq}, 1}=1+\sum_{k=2}^{k_{\max }}\left(1-e^{-\frac{\Delta t_{\mathrm{k}, \mathrm{k}-1}}{\tau_{\mathrm{rec}}}}\right) .
$$




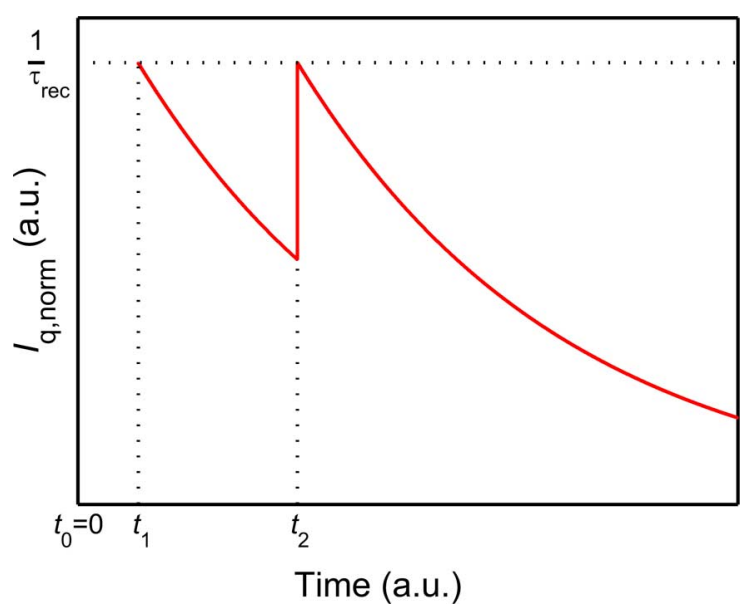

Fig. 4. Example of the normalized current pulse through the quench resistor of a microcell induced by two discharges triggered at times $t_{1}$ and $t_{2}$.

It is noted that the normalized single microcell signal shape at the output of the SiPM might in fact differ somewhat from $I_{\mathrm{q}, \text { norm }}$, as it is shaped due to the various capacitances and inductances, which might not be lossless, in the SiPM [29], [30]. However, assuming that microcells do not influence each other, the total integrated signal of all cells at the output of the SiPM, $N_{\mathrm{f}, \text { eq }}$, expressed in terms of the equivalent number of fired cells, simply equals the sum of all $N_{\mathrm{f}, \mathrm{eq}, 1}$.

3) Afterpulsing: During each discharge, there is a possibility that one or more traps in the microcell are occupied by charge carriers from the discharge. These may subsequently be released and trigger an additional discharge. This phenomenon is referred to as afterpulsing. The probability distribution $p_{\text {nap }}$ of the number of afterpulses originating from a single discharge can be described as a Poisson distribution with expectation value $\bar{N}_{\text {ap }}$ [32].

For each trapped charge carrier that initiates an additional discharge, the probability distribution $p_{\mathrm{ap}}(\Delta t)$ of the elapsed time $\Delta t$ between the triggering of the discharge causing the afterpulse and the triggering of the afterpulse itself can be described as a sum of multiple exponentials with time constants ranging from $\sim 10^{-8}$ to $\sim 10^{-5} \mathrm{~s}$ [33]. However, the filled trap density corresponding to the smallest release time constant is at least $\sim 2$ times larger than each of the other filled trap densities. Furthermore, when measuring the charge content of SiPM pulses, a time window is often applied with the result that afterpulses occurring a relatively long time after the primary trigger are not recorded. For these reasons, only a single trapped-carrier release time constant $\tau_{\text {ap }}$ is taken into account here

$$
p_{\text {ap }}(\Delta t)= \begin{cases}\frac{1}{\tau_{\mathrm{ap}}} e^{-\frac{\Delta t}{\tau_{\mathrm{ap}}}}, & \Delta t \geq 0 \\ 0, & \Delta t<0 .\end{cases}
$$

It should be noted that a weighted sum of exponentials could relatively easily be implemented in the SiPM response model when necessary. The effect of saturation on afterpulsing and how to correct for it are discussed in Section III-C.

4) Optical Crosstalk: Another process, which may occur within a cell during a discharge, is the emission of optical photons that may reach other cells and trigger additional discharges
[34], [35]. This process is referred to as optical crosstalk and is considered to be instantaneous. The probability distribution $p_{\text {oct }}$ of the number of crosstalk-induced triggers due to a single primary trigger, including the possible cascade of crosstalk inducing other crosstalk, is assumed to be Poisson-distributed with expectation value $\bar{N}_{\text {oct }}$. Then, if we neglect saturation and crosstalk following afterpulsing, the probability distribution $p_{\text {oct, }, 1}$ of the number of crosstalk-induced triggers in any given cell due to $\bar{N}_{\mathrm{pt}, 1}$ primary triggers in each of the other $N_{\text {cells }}-1$ cells is Poisson-distributed with expectation value

$$
\bar{N}_{\mathrm{oct}, 1} \approx \bar{N}_{\mathrm{oct}}\left(1+\bar{N}_{\mathrm{oct}}\right) \bar{N}_{\mathrm{pt}, 1} .
$$

The sum of the number of primary triggers and the number of crosstalk-induced triggers is referred to as the number of photon-induced triggers in the remainder of this document. The effects of saturation and afterpulsing on crosstalk are discussed in Section III-C.

5) Dark Counts: Thermally released charge carriers in the breakdown region of a microcell may give rise to discharges, which are referred to as dark counts. The expectation value of the output charge due to dark counts $\bar{N}_{\mathrm{dc}}$, expressed in terms of the equivalent number of fired cells, can be calculated as the product of the expectation value of the dark count rate $r_{\mathrm{dc}}$ and the integration time window $\Delta t_{\text {meas }}$ used in a measurement

$$
\bar{N}_{\mathrm{dc}}=r_{\mathrm{dc}} \Delta t_{\text {meas }} .
$$

Usually, $\Delta t_{\text {meas }}$ will be of the order of the output signal duration.

When the average time between two dark counts $r_{\mathrm{dc}}^{-1}$ is much larger than $\tau_{\mathrm{d}}, \tau_{\text {rec }}$, and $\tau_{\text {ap }}$, the influence of dark counts on the response is negligible, as is the case for the measurements in this work; see Section V-A. In case of a very slow scintillation signal, i.e., when $r_{\mathrm{dc}}^{-1}$ is much smaller than $\tau_{\mathrm{d}}, \tau_{\mathrm{rec}}$, and $\tau_{\mathrm{ap}}$, the response is not expected to suffer from saturation. In that case, the influence of $\bar{N}_{\mathrm{dc}}$ can simply be modeled as an offset to the response, where it should be noted that dark counts may also induce afterpulses and crosstalk. Finally, in cases where $r_{\mathrm{dc}}^{-1}$ is of the order of $\tau_{\mathrm{d}}, \tau_{\mathrm{rec}}$, or $\tau_{\mathrm{ap}}$, the model would require an extension to include dark counts and their cross correlation with the other effects.

\section{SiPM Response to Scintillation Pulses}

During a scintillation pulse, many different sequences of the various events described in the previous section are possible in each microcell. In order to calculate the SiPM response, we first calculate the expected output charge of a single cell for a fixed number of photon-induced triggers $i$ and for fixed numbers of afterpulses $j, k, \ldots$ for each photon-induced trigger. Then, using the probability of each possible sequence, the expected output charge of a single cell is calculated, from which the SiPM response is finally obtained. The output charge is henceforth expressed in terms of the equivalent number of fired cells, unless mentioned otherwise.

1) Single-Microcell Output Charge: We define $\psi_{1, j}$ as the expectation value of the output charge of a single cell, in the case of a single photon-induced trigger, giving rise to $j$ afterpulses. Similarly, $\psi_{2, j, k}$ denotes the expectation value of the 
output charge of a single cell, in the case of two photon-induced triggers in that cell, giving rise to $j$ and $k$ afterpulses, respectively. For each number of photon-induced triggers $i>2$, the expectation value of the output charge of a single cell, giving rise to fixed numbers of afterpulses for each photon-induced trigger, can be denoted in an equivalent way.

$\psi_{i, j}, \psi_{i, j, k}, \ldots$ are functions of $i, \tau_{\mathrm{d}}, \tau_{\mathrm{rec}}, \tau_{\mathrm{ap}}$, and the numbers of afterpulses $j, k, \ldots$. They take into account the temporal dependencies of the scintillator decay, the SiPM recovery, and the afterpulsing, each of which were discussed in Section III-B. The derivations of $\psi_{i, j}, \psi_{i, j, k}, \ldots$ are given in Appendix A. For reasons of simplicity, it is assumed that the probability of afterpulsing is small enough that afterpulses induced by afterpulses can be neglected. If the number of photon-induced triggers $i=0$, there is no output charge and therefore $\psi_{0}=0$.

The probability of the number of afterpulses for each photoninduced trigger is Poisson-distributed with probability distribution $p_{\text {nap }}$ (see III-B3). We assume that the number of charge carriers trapped during a discharge is proportional to the total number of charge carriers in the discharge. Then, due to the finite recovery time of each cell, the average number of afterpulses for each photon-induced trigger occurring in a scintillation event is smaller than the average number of afterpulses $\bar{N}_{\text {ap }}$ occurring upon a single discharge. Therefore, the probability distributions $p_{\text {nap }, i}$ of the number of afterpulses of each photon-induced trigger, for $i$ photon-induced triggers occurring in a single cell, can be described as a Poisson distribution with expectation values $\bar{N}_{\text {ap }, i}$ calculated by multiplying $\bar{N}_{\text {ap }}$ with the average relative amount of charge per photon-induced trigger

$$
\bar{N}_{\mathrm{ap}, 1}=\bar{N}_{\mathrm{ap}} \frac{\psi_{1,0}}{1}, \quad \bar{N}_{\mathrm{ap}, 2}=\bar{N}_{\mathrm{ap}} \frac{\psi_{2,0,0}}{2}, \quad \bar{N}_{\mathrm{ap}, 3}=\ldots
$$

Then, $\xi_{1}$, which we define as the expectation value of the output charge of a single cell in the case of one photon-induced trigger, is calculated as the expectation value of $\psi_{1, j}$

$$
\xi_{1}=\sum_{j=0}^{\infty} p_{\text {nap }, 1}(j) \psi_{1, j}
$$

Similarly, the output charge of a single cell in the case of two photon-induced triggers, $\xi_{2}$, is calculated as the expectation value of $\psi_{2, j, k}$

$$
\xi_{2}=\sum_{j=0}^{\infty} \sum_{k=0}^{\infty} p_{\text {nap }, 2}(j) p_{\text {nap }, 2}(k) \psi_{2, j, k} .
$$

For $i>2, \xi_{i}$ can be calculated in an equivalent way.

2) Numerical Computation of the Single-Microcell Output Charge: The calculation of $\psi_{1, j}, \psi_{2, j, k}, \ldots$ described in Appendix A results in series that grow rapidly in size with the number of photon-induced triggers $i$ and the corresponding numbers of afterpulses, leading to long computation times. In the remainder of this work it is therefore assumed that the average number $\bar{N}_{\text {ap }}$ of afterpulses due to a single fired microcell is less than $\sim 0.5$. Then, in order for the cumulative distribution function (cdf) of the number of afterpulses to reach $>99 \%, \xi_{i}$ only has to be calculated for up to three afterpulses for each absorbed photon.

If it is furthermore assumed that there are on average less than $\sim 4$ photon-induced triggers per microcell per light pulse, $\chi$ needs to be calculated for up to nine photon-induced triggers per microcell only in order for the cdf of the number of photon-induced triggers to reach $>99 \%$. When working with scintillation detectors, this assumption is often satisfied as excessive saturation is usually avoided. It should nevertheless be noted that for $i>9$, the numerical computation of $\xi_{i}$ requires so much time that it may be more practical to obtain additional terms by fitting and extrapolating $\xi_{i}$ as a function of $i$ as demonstrated in Appendix B.

3) SiPM Output Charge and Response: Assuming, for the moment, that the scintillation photons are homogenously distributed over the SiPM, the expectation value of the total output charge of a single cell $\bar{N}_{\mathrm{f}, \mathrm{eq}, 1}$ in response to a scintillation event can be obtained as follows. A scintillator with absolute light yield $Y$ is considered, in which a gamma photon with energy $E_{\gamma}$ is absorbed, so that the number of created scintillation photons at the photopeak position equals $N_{\mathrm{ph}}=E_{\gamma} Y$. A fraction $f$ of these photons is absorbed in one or more cells of the SiPM and may trigger discharges. Thus, $f$ depends on the crystal aspect ratio, reflective materials, losses within the crystal, etc. Provided that $\varepsilon / N_{\text {cells }} \ll 1$ and $f \cdot N_{\text {ph }} \gg 1$, the probability distribution $p_{\mathrm{pt}, 1}$ of the number of such primary triggers in any cell is Poisson distributed with expectation value

$$
\bar{N}_{\mathrm{pt}, 1}=\frac{\varepsilon}{N_{\text {cells }}} f N_{\mathrm{ph}}
$$

Taking into account crosstalk as discussed in Section III-B4, where it was assumed that the influence of saturation and afterpulsing on crosstalk is negligible, the probability distribution $p_{\text {pit,1 }}(i)$ of the number of photon-induced triggers $i$ in any cell can be written as a Poisson distribution with expectation value

$$
\bar{N}_{\mathrm{pit}, 1} \approx \bar{N}_{\mathrm{pt}, 1}+\bar{N}_{\mathrm{oct}}\left(1+\bar{N}_{\mathrm{oct}}\right) \bar{N}_{\mathrm{pt}, 1}
$$

Here, use has been made of (9).

The total output charge of a single cell, $\bar{N}_{\text {f,eq, } 1}$, can now be calculated as the expectation value of $\xi_{i}$

$$
\bar{N}_{\mathrm{f}, \mathrm{eq}, 1}=\sum_{i=1}^{\infty}\left[p_{\mathrm{pit}, 1}(i) \cdot \xi_{i}\right]
$$

However, the number of crosstalk photons being released during a discharge is proportional to the number of electrons in the discharge [34]. Hence, the average amount of crosstalk per photon-induced trigger due to a scintillation event differs from that due to a single fired cell because of saturation and afterpulsing. A simple, first-order correction on $\bar{N}_{\text {pit }, 1}$ can be obtained by multiplying $\bar{N}_{\text {oct }}$ with an approximation of the average charge per photon-induced trigger, viz. $\xi_{\mathrm{Npit}, \text { linint }} / \bar{N}_{\mathrm{pit}, 1}$, where $\xi_{\text {Npit, linint }}$ is linearly interpolated between the $\xi_{i}$ corresponding to the two values of $i$ closest to $\bar{N}_{\text {pit }, 1}$. 
In this work, a more accurate corrected expectation value of the number of photon-induced triggers in any cell is used, as follows:

$$
\begin{aligned}
\bar{N}_{\mathrm{pit}, 1, \mathrm{cor}} \approx & \bar{N}_{\mathrm{pt}, 1} \\
& +\bar{N}_{\mathrm{oct}} \frac{\bar{N}_{\mathrm{f}, \mathrm{eq}, 1}}{\bar{N}_{\mathrm{pit}, 1, \mathrm{cor}}}\left(1+\bar{N}_{\mathrm{oct}} \frac{\bar{N}_{\mathrm{f}, \mathrm{eq}, 1}}{\bar{N}_{\mathrm{pit}, 1, \mathrm{cor}}}\right) \bar{N}_{\mathrm{pt}, 1}
\end{aligned}
$$

which in combination with (16) is solved iteratively. It should be noted that crosstalk following afterpulses is included in the crosstalk due to primary triggers, resulting in a small overestimation of the number of photon-induced triggers and also in a small underestimation of the contribution of afterpulses to the total output charge.

Finally, since the expectation value $\bar{N}_{\mathrm{f} \text {,eq }}$ of the total output charge of the SiPM simply equals the product of $N_{\text {cells }}$ and $\bar{N}_{\text {f,eq,1 }}$, the SiPM response $\chi$ can be expressed as

$$
\chi=\frac{N_{\mathrm{cells}} \bar{N}_{\mathrm{f}, \mathrm{eq}, 1}}{\varepsilon f N_{\mathrm{ph}}} .
$$

In cases where it cannot be assumed that the scintillation photons are homogenously distributed over the SiPM, a number of partial responses $\chi_{k}$ may be defined for $n$ groups of $N_{\text {cells, } k}$ microcells, over each of which a fraction $f_{k}$ of the scintillation photons can be assumed to be absorbed homogenously. For each $k \leq n, \chi_{k}$ can then be calculated using (18), where $f$ is re-

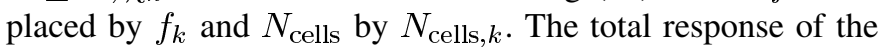
$\mathrm{SiPM}$ can then be calculated as the weighted average of the partial responses

$$
\chi=\sum_{k=1}^{n} \frac{f_{k}}{f} \chi_{k}
$$

where the number of groups $n$ is an integer between 1 and $N_{\text {cells }}$. For simplicity, it is assumed that the scintillation photons are homogenously distributed over the cells in the remainder of this work.

\section{SiPM Response to Fast Light Pulses}

If the light pulse is much shorter than the SiPM recovery time, it can be considered instantaneous. Then, if during a discharge a second photon triggers a discharge in the same cell, the output charge of that cell corresponds to only one discharge. Therefore, in case of a fast light pulse, the expectation value of the output charge of a single cell $\bar{N}_{\text {f,eq, } 1 \text {,fast }}$ simplifies from (16) to

$$
\bar{N}_{\mathrm{f}, \mathrm{eq}, 1, \mathrm{fast}}=\sum_{i=1}^{\infty}\left[p_{\mathrm{pit}, 1}(i)\right] \cdot \xi_{1}=\left(1-p_{\mathrm{pit}, 1}(0)\right) \cdot \xi_{1}
$$

where it is assumed that crosstalk following afterpulses can be neglected. The response $\chi_{\text {fast }}$ for fast light pulses then simplifies from (14), (15), (18), and (20) to

$$
\chi_{\text {fast }}=\frac{N_{\text {cells }}}{\varepsilon f N_{\mathrm{ph}}} \times\left(1-e^{-\left[1+\bar{N}_{\mathrm{oct}}\left(1+\bar{N}_{\mathrm{oct}}\right)\right] \frac{\varepsilon f N_{\mathrm{ph}}}{N_{\mathrm{cells}}}}\right) \xi_{1}
$$

where $\xi_{1}$ can be simplified from (12) and from (29) in Appendix A to

$$
\xi_{1}=\sum_{j=0}^{\infty} \frac{\bar{N}_{\mathrm{ap}}^{j}}{j !} e^{-\bar{N}_{\mathrm{ap}}}\left\{(1+j)-\sum_{k=1}^{j} \frac{1}{1+\frac{\tau_{\mathrm{ap}}}{k \tau_{\mathrm{rec}}}}\right\}
$$

where $k$ is an integer used for summing. It should be noted that $\chi_{\text {fast }}$ calculated in (21) equals $\chi_{\text {lower }}$ described by the simple exponential model in (3) if there is no afterpulsing and crosstalk, i.e., if $\bar{N}_{\text {oct }}=0$ and $\xi_{1}=1$.

\section{MATERIALs, ExPERIMENTS, AND Methods}

The SiPM response model is validated with measurements using two SiPMs type Hamamatsu MPPC S10362-33-050C $\left(3 \times 3 \mathrm{~mm}^{2}\right.$ active area with $50 \times 50 \mu \mathrm{m}^{2}$ microcells [36]), henceforth referred to as detector 1 and detector 2 . All measurements were performed at a temperature of $22 \pm 1^{\circ} \mathrm{C}$ at the nominal bias voltages specified by the manufacturer. The response of the SiPMs was measured as function of $N_{\mathrm{ph}}$ and compared to the modeled response using measured parameters of our specific devices as input for the model.

\section{A. SiPM Parameter Measurements}

The voltage-over-breakdown $V_{\mathrm{ob}}$ was determined as described by Seifert et al. [29]. The value $R_{\mathrm{q}}$ of the quench resistors was determined by forward-biasing the SiPM and measuring the IV-curve.

The SiPM gain $M$, i.e., the average output charge due to a single fired cell, was determined as follows. The SiPM output current signal was converted by a shunt resistor $R_{\mathrm{S}}=15 \Omega \pm$ $0.15 \Omega$ into a voltage, which was amplified by a noninverting voltage amplifier based on a TI OPA847 operational amplifier, providing a gain of 13.27. The amplified signals were sampled with an Acqiris DC282 fast 10-bit digitizer at a sampling rate of $8 \mathrm{GS} / \mathrm{s}$. In each measurement, the input voltage range of the digitizer was set for an optimal use of the dynamic range of the digitizer so as to minimize the ADC noise. The sampling time window of 500 ns was chosen to match the length of the SiPM pulses.

The SiPM was illuminated with a Hamamatsu PLP-04 laser (wavelength $633 \mathrm{~nm}$, average pulse duration $50 \mathrm{ps}$, repetition rate $10 \mathrm{kHz}$ ). The intensity of the light was regulated with neutral density filters such that, on average, $\sim 30$ photons reached the SiPM. The laser and the digitizer were triggered by the same clock generator.

The average pulse shape was determined by recording 50000 traces, correcting them for offsets, normalizing them to the same peak height, and averaging them. The resulting averaged pulse was again corrected for offset so as to remove the homogeneous contribution of dark counts. It is emphasized that the averaged pulse is influenced by afterpulsing, resulting in a larger pulse area. This was corrected by calculating and subtracting the contribution of afterpulsing by means of the response model.

Subsequently, an additional neutral density filter was added, such that on average only a few photons reached the SiPM. Again, 50000 traces were recorded and corrected for offset. All of these traces were fitted with the averaged pulse using the peak 
height as the fitting parameter. The obtained peak heights were multiplied with the corrected area of the averaged pulse, and a histogram was created. The resulting comb structure was fitted with a sum of Gaussians, where the distance between the peak positions was set to be constant. This distance is a measure of the gain observed at the output of the preamplifier, expressed in $\mathrm{V} \cdot \mathrm{s}$. The SiPM gain $M$ for both devices was subsequently calculated by correcting for the preamplifier gain and dividing by the shunt-resistor value $R_{\mathrm{S}}$ and the elementary charge $e$.

The recovery time $\tau_{\text {rec }}$ could then be calculated by inserting (1) into (6)

$$
\tau_{\text {rec }}=\frac{e M}{V_{\mathrm{ob}}} R_{\mathrm{q}} \text {. }
$$

The SiPM parameters $\bar{N}_{\text {oct }}, \bar{N}_{\text {ap }}, \tau_{\text {ap }}$, and $r_{\text {dc }}$ were obtained in a similar way as described by Du and Retière [32]. For convenience, the method is briefly summarized, and differences and additions are described where necessary. Both SiPMs were connected to a transistor-based, high-bandwidth, low-noise amplifier with a gain of $\sim 50$. The amplified signals were sampled with an Acqiris DC282 fast 10-bit digitizer at a sampling rate of $4 \mathrm{GS} / \mathrm{s}$.

The average pulse shape was determined in the same way as described for the determination of $M$. Then, the laser was removed, and 50000 random traces were recorded per detector using a time window of $2.5 \mu \mathrm{s}$. All traces were analyzed using a pulse-finding method that worked in four steps. It located the first pulse by comparing the trace values to a certain threshold. Then, the pulse was fitted with either one or the sum of two average pulses, depending on the rise time and on the number of maxima of the pulse. Here, the starting time(s) of the pulse(s) and amplitude(s) were the fitting parameters. Finally, the starting time(s) and the corresponding amplitude(s) were stored, and the fit was subtracted from the trace. This was repeated until no more pulses were found in the trace.

For all pulses, the time difference with the preceding pulse was calculated and stored together with its amplitude. To exclude afterpulses from earlier discharges, only those pulses were used for which the $300 \mathrm{~ns}$ before the preceding pulse contained no other pulses. Furthermore, only those pulses were used for which the amplitude of the preceding pulse corresponded to a single discharge.

From the resulting amplitude-versus-time-difference diagram (showing a similar horizontal band structure of amplitudes corresponding to $1,2,3, \ldots$ fired cells as presented by $\mathrm{Du}$ and Retière [32]), the probability $P_{1 \text {,oct }}$ that one fired cell triggers exactly one other cell was determined from the fraction of pulses $f_{1, \text { oct }}$ with exactly one crosstalk-induced trigger (i.e., the band of amplitudes corresponding to two fired cells). Given this definition of $P_{1 \text {,oct }}$, which is different from the way crosstalk is characterized by Du and Retière [32], one needs to take into account that the same fraction $f_{1 \text {,oct }}$ of the single crosstalk-induced triggers also induces crosstalk

$$
P_{1, \mathrm{oct}}=f_{1, \mathrm{oct}} \sum_{k=0}^{\infty} f_{1, \mathrm{oct}}^{k}=\frac{f_{1, \mathrm{oct}}}{1-f_{1, \mathrm{oct}}} .
$$

For the determination of $f_{1 \text {,oct }}$, only time differences larger than $200 \mathrm{~ns}$ were taken into account since crosstalk originating from afterpulses cannot be separated from dark counts in the amplitude-versus-time-difference diagram.

The expectation value $\bar{N}_{\text {oct }}$ of the number of crosstalk-induced triggers was subsequently calculated as

$$
\bar{N}_{\text {oct }}=\sum_{k=1}^{\infty} k 2^{k-1}\left(P_{1, \text { oct }}\right)^{k}=\frac{\left(1-f_{1, \text { oct }}\right) f_{1, \text { oct }}}{\left(1-3 f_{1, \text { oct }}\right)^{2}}
$$

where the factor $2^{k-1}$ accounts for the possibilities to induce $k$ discharges including crosstalk-induced crosstalk.

In addition, a histogram of the time differences of the selected set of recorded pulses was created and normalized to the area of the histogram. The parameters $\bar{N}_{\mathrm{ap}}, \tau_{\mathrm{ap}}$ and $r_{\mathrm{dc}}$ could then be determined by fitting the histogram with a function $P(t)$, which, because of a misprint in [32], is repeated in its correct form here:

$$
\begin{aligned}
P(t)= & \left(1-\int_{0}^{t} P_{\mathrm{AP}}(x) \mathrm{d} x\right) P_{\mathrm{DN}}(t) \\
& +\left(1-\int_{0}^{t} P_{\mathrm{DN}}(x) \mathrm{d} x\right) P_{\mathrm{AP}}(t)
\end{aligned}
$$

where $P_{\mathrm{AP}}(t)$ and $P_{\mathrm{DN}}(t)$ are the probabilities that the first afterpulse or dark count, respectively, occurs in the time interval $t$ and $t+\mathrm{d} t$ after the starting pulse.

\section{B. SiPM Response Measurements}

Measurements of the SiPM response were performed using two $3 \times 3 \times 5 \mathrm{~mm}^{3} \mathrm{LaBr}_{3}: 5 \%$ Ce scintillators optically coupled to the SiPMs, using a Silicone encapsulation gel (Lightspan LS-3252). All crystal faces not coupled to the SiPM were covered with a highly reflective PTFE-based material (Spectralon). Since $\mathrm{LaBr}_{3}: 5 \% \mathrm{Ce}$ has a high light yield (70 ph/keV at $662 \mathrm{keV}$ [37]) and a fast decay time $\left(\tau_{\mathrm{d}}=16 \mathrm{~ns}\right.$ [38]), significant saturation of the SiPM is expected, resulting in a clear dependency of the response on the number of emitted photons. The SiPM signals were amplified and digitized in the same way as described for the determination of $M$ in the previous section, except that a sampling rate of $4 \mathrm{GS} / \mathrm{s}$ was used.

Pulse height spectra were recorded for several isotopes, i.e., I-125, Am-241, Hg-203, Na-22, Cs-137, Y-88, and Co-60, emitting gamma photons at 10 different energies between 27.3 and $1836 \mathrm{keV}$. The numbers of emitted photons $N_{\mathrm{ph}}$ corresponding to 27.3 and $59.5 \mathrm{keV}$ were calculated taking into account the light yield nonproportionality of $\mathrm{LaBr}_{3}$ :Ce with values of 0.964 and 0.986, respectively [39]. Between 10000 and 50000 pulses were recorded for each isotope. The pulses were corrected for offset by subtracting the mean value of the 25 data points preceding the pulse and were subsequently integrated. In order to express the resulting values in terms of the equivalent number of fired cells, they were divided by the gain $M$ determined in the previous section. A histogram was created, and the position of the photopeak was determined by fitting it with a Gaussian. Due to the good energy resolution of $\mathrm{LaBr}_{3}$ : $\mathrm{Ce}(\sim 3 \% \mathrm{FWHM}$ at $662 \mathrm{keV}$ ) [40], it can be assumed that the SiPM response only affects the photopeak position while negligibly affecting its shape. 
TABLE I

MeAsured SiPM Parameter Values of THE two Hamamatsu MPPC S10362-33-050C DeVICES. All UnCERTAINTIES QUOTED ARE 95\% CONFIDENCE INTERVALS, BASED EITHER ON FITS OR ON ESTIMATED MEASUREMENT UNCERTAINTIES

\begin{tabular}{|c|c|c|c|c|c|c|c|c|}
\hline Detector & $\begin{array}{l}V_{\mathrm{ob}} \\
(\mathrm{V})\end{array}$ & $\begin{array}{c}R_{\mathrm{q}} \\
(\mathrm{k} \Omega)\end{array}$ & $\begin{array}{c}M \\
\left(10^{5}\right) \\
\end{array}$ & $\begin{array}{c}\tau_{\text {rec }} \\
(\mathrm{ns})\end{array}$ & $\bar{N}_{\mathrm{ap}}$ & $\begin{array}{c}\tau_{\mathrm{ap}} \\
(\mathrm{ns})\end{array}$ & $\begin{array}{c}r_{\mathrm{dc}} \\
(\mathrm{MHz})\end{array}$ & $\bar{N}_{\text {oct }}$ \\
\hline Detector 1 & $1.26 \pm 0.05$ & $145.3 \pm 0.5$ & $8.3 \pm 0.5$ & $15.3 \pm 1$ & $0.124 \pm 0.005$ & $25 \pm 8$ & $5.6 \pm 0.1$ & $0.140 \pm 0.005$ \\
\hline Detector 2 & $1.39 \pm 0.05$ & $140.4 \pm 0.5$ & $7.9 \pm 0.5$ & $12.8 \pm 1$ & $0.132 \pm 0.005$ & $26 \pm 8$ & $5.1 \pm 0.1$ & $0.132 \pm 0.005$ \\
\hline
\end{tabular}

The output charge corresponding to the photopeak position was subsequently corrected for the gain nonproportionality described in Section II-A. To this end, the voltage-over-breakdown $V_{\mathrm{ob}}(t)$ as a function of time was estimated by subtracting the average output pulse corresponding to the photopeak position from the undisturbed $V_{\mathrm{ob}}$. By multiplying the calculated $V_{\mathrm{ob}}(t)$ with the probability distribution of the scintillation pulse $p_{\mathrm{sc}}(t)$ and integrating this product, a weighted average of $V_{\mathrm{ob}}(t)$ over the duration of the scintillation pulse was calculated. This weighted average was divided by the value of the undisturbed $V_{\mathrm{ob}}$, yielding the relative gain nonproportionality. It should be noted that in case of a slower or lower light yield scintillator, it may be required to perform this correction on an event-per-event basis.

\section{Detector PDE Determination}

To calculate the SiPM responses from the measurements and from the model, and to compare them as a function of $N_{\mathrm{ph}}$, the value of the detector PDE, $\varepsilon \cdot f$, needs to be determined. Since the detector PDE depends on the detector geometry, the scintillator, the use of reflecting materials, etc., it is difficult to measure it directly. Therefore, the value of $\varepsilon \cdot f$ was chosen so as to match the modeled and the measured response at the measurement that is least influenced by saturation, i.e., the one at $27.3 \mathrm{keV}$, corresponding to 1842 emitted photons. Here, literature values of the absolute light yield of $\mathrm{LaBr}_{3}$ :Ce have been used [37], [39]. It should be noted that the model requires the product of $N_{\mathrm{ph}}$ and $\varepsilon \cdot f$ as an input. Therefore, in case one uses the model to derive an accurate value of the detector PDE, also the absolute light yield needs to be determined accurately.

\section{RESULTS AND DISCUSSION}

\section{A. SiPM Parameters Measurements}

The measured voltage-over-breakdown $V_{\mathrm{ob}}$, quench resistor value $R_{\mathrm{q}}$, SiPM gain $M$, and SiPM recovery time $\tau_{\text {rec }}$ are given in Table I. The corrections on $M$ due to afterpulsing were equal to factors of 1.076 and 1.087 for detectors 1 and 2, respectively. The values of $M$ are in good correspondence with those in the data sheet of the manufacturer for the $1 \times 1 \mathrm{~mm}^{2}$ version of the same type of SiPM (Hamamatsu MPPC S10362-11-050C) at the same temperature [41].

The measurements for the determination of $\bar{N}_{\text {oct }}, \bar{N}_{\text {ap }}, \tau_{\text {ap }}$, and $r_{\mathrm{dc}}$ (see Section IV-A) yielded an amplitude-versus-timedifference diagram and a time-difference histogram with similar features as those presented by Du and Retière [32]. The derived parameter values are given in Table I.

The values of $\bar{N}_{\text {ap }}$ and $\tau_{\text {ap }}$ are very similar to the values obtained by $\mathrm{Du}$ and Retière for the $1 \times 1 \mathrm{~mm}^{2}$ version of the

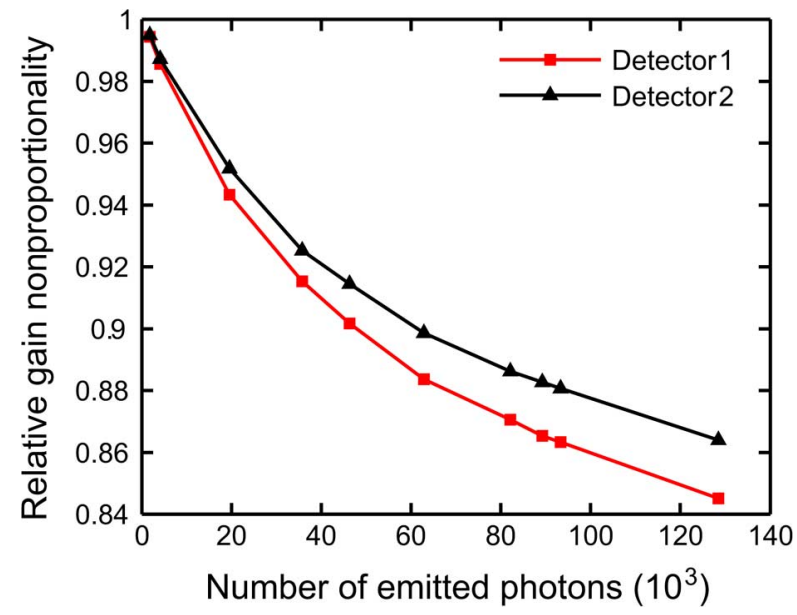

Fig. 5. Estimated relative gain nonproportionality as a function of the number of emitted photons $N_{\mathrm{ph}}$ for both SiPMs investigated. The line segments connecting the symbols are drawn to guide the eye.

same type of SiPM (Hamamatsu MPPC S10362-11-050C), viz. $\sim 0.13$ for $\bar{N}_{\text {ap }}$ and $\sim 15 \mathrm{~ns}$ for $\tau_{\text {ap }}$ [32]. However, it is emphasized that the determination of $\bar{N}_{\text {ap }}$ and $\tau_{\text {ap }}$ may be influenced by the pulse-finding method and by the range used for fitting the time-difference histogram.

The dark count rates are close to the value of $6 \mathrm{MHz}$ at $25^{\circ} \mathrm{C}$ specified by the manufacturer [36]. This means that a dark count occurs on average every $\sim 180 \mathrm{~ns}$, which is much longer than $\tau_{\mathrm{d}}$. Hence, dark counts can indeed be neglected.

Values for $f_{1, \text { oct }}$ of 0.085 and 0.082 were obtained for detectors 1 and 2, respectively. The respective probabilities that one fired cell triggers at least one other cell, i.e., 0.093 and 0.089, are very similar to the values obtained by Du and Retière for the $1 \times 1 \mathrm{~mm}^{2}$ version of the same type of SiPM (Hamamatsu MPPC S10362-11-050C), viz. 0.09 [32].

\section{B. SiPM Response Measurements}

The relative SiPM gain nonproportionality was estimated for the different gamma energies used in the pulse height measurements. The result is shown in Fig. 5.

It can be seen that larger energies, which induce larger SiPM signals, result in a larger temporary reduction of the voltage-over-breakdown. It should be noted that, due to saturation, the weight of the first part of the photon pulse might be relatively larger, resulting in an uncertainty of the relative gain nonproportionality.

All pulse height spectra were corrected for the estimated gain nonproportionality. Fig. 6 shows the resulting pulse heights as 


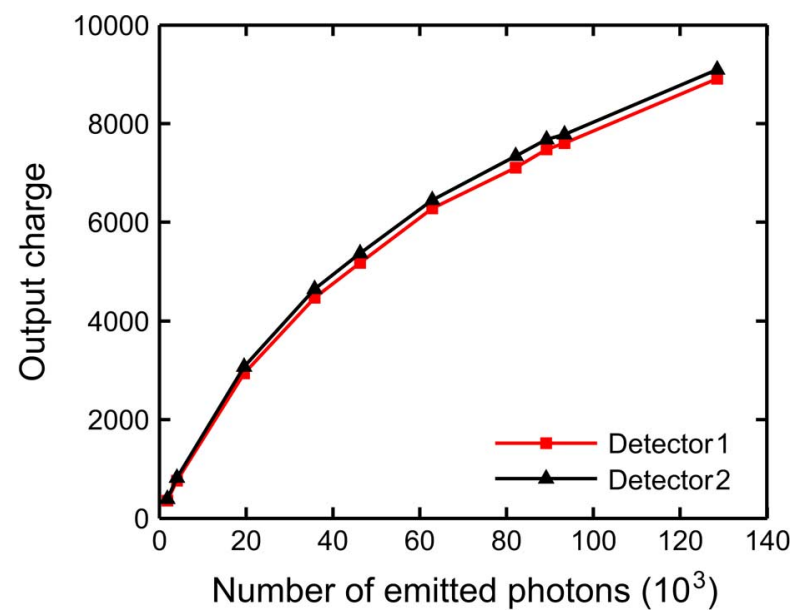

Fig. 6. The measured expectation value of the SiPM output charge, expressed in terms of the equivalent number of fired cells $\bar{N}_{\mathrm{f} \text {,eq }}$ as a function of the number of scintillation photons $N_{\mathrm{ph}}$, for both SiPMs investigated. The values are corrected both for gain nonproportionality and for scintillator nonproportionality. The line segments connecting the symbols are drawn to guide the eye.

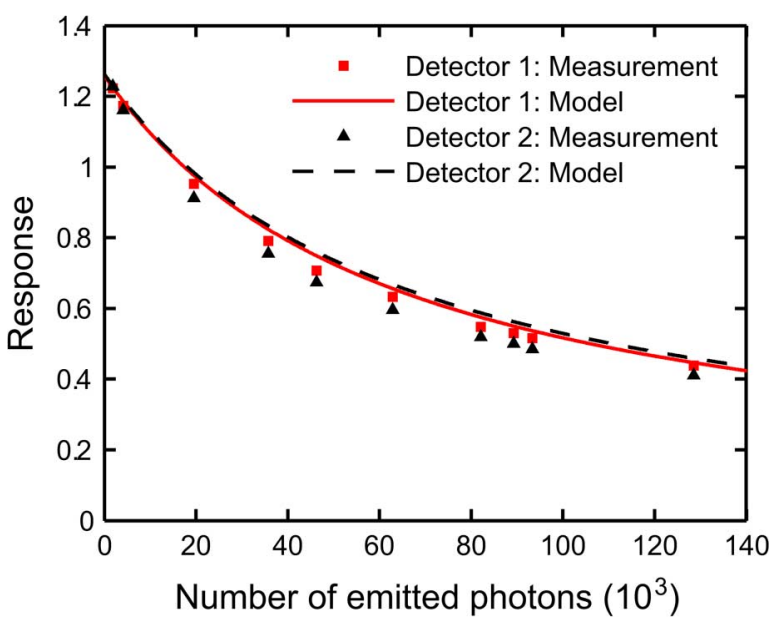

Fig. 7. The measured (symbols) and the modeled (curves) responses of the two SiPMs coupled to two $\mathrm{LaBr}_{3}$ :Ce crystals.

a function of the number of scintillation photons $N_{\text {ph }}$ per event, for both SiPMs.

\section{Model Validation}

The values of the detector PDE of the SiPM-based detectors used in this work were determined to be 0.16 and 0.17 for detectors 1 and 2, respectively.

Using these values and the parameters measured in Section V-A, the response of the two SiPMs as a function of $N_{\mathrm{ph}}$ was predicted with the model. To this end, $\xi_{i}$ was calculated for $1 \leq i \leq 9$ using the method described in Section III-C and Appendix A. However, the expectation value $\bar{N}_{\text {pid,1 }}$ of the number of photon-induced triggers in any cell is $\sim 7$ for the largest gamma energy used in the measurements, viz. $1836 \mathrm{keV}$. Therefore, in order for the cumulative distribution function of the number of photon-induced triggers per cell to reach $>99 \%$, the values of $\xi_{i}$ were fitted and extrapolated to $i=14$ as described in Appendix B. Fig. 7 shows both the modeled and the measured response. Good agreement is observed.
There are a number of potential reasons for the remaining discrepancies. They can at least partly be attributed to the uncertainties in the many different measurements performed to determine the various interdependent parameters, including the correction of the gain nonproportionality. The largest contribution to the overall uncertainty is in fact expected from the measurement of $M$, which involves measuring the small signals resulting from only a few photons. Because of this and the relatively large device capacitance, noise causes the determination of $M$ to be difficult. Furthermore, small temperature differences between the gain determination measurement and the SiPM response measurement could have contributed to an uncertainty of $M$. These uncertainties of $M$ could indirectly affect the uncertainty in $\varepsilon \cdot f$ and $\tau_{\text {rec }}$ as well. It should be noted that the measurements at the lowest gamma energy, i.e., $27.3 \mathrm{keV}$, are of large significance to both the modeled and the measured response, since these are used for the determination of $\varepsilon \cdot f$.

A second potential cause of uncertainty concerns the assumption of an instantaneous discharge process. In this simplification the probability is neglected that during the quenching of the discharge, i.e., during the rise time of the current signal through the quench resistor, additional triggers might occur, which do not contribute to the output charge. The rise time of the current signal through the quench resistor can thus be seen as a dead time. The effect of this dead time will be more pronounced when triggers occur more closely together in time. This might result in a small overestimation of the response for the measurements at the higher gamma energies.

\section{Model Comparison to Known Cases}

The SiPM response was predicted as a function of $N_{\mathrm{ph}}$ for three different cases for which the behavior of the response is known a priori, viz. for a scintillator decay time much longer than $\tau_{\text {rec }}$ and $\tau_{\text {ap }}$, i.e., $\tau_{\mathrm{d}}=10 \mu$ s; for an instantaneous light pulse without afterpulsing and crosstalk, i.e., $\tau_{\mathrm{d}}=0, \bar{N}_{\text {oct }}=0$, and $\bar{N}_{\text {ap }}=0$; and for an instantaneous light pulse including afterpulsing and crosstalk. All other SiPM parameters as well as $\varepsilon \cdot f$ were kept equal to the values determined for detector 1 . The result is presented in Fig. 8 in comparison to the simple exponential model given by (3).

The response for $\tau_{\mathrm{d}}=10 \mu \mathrm{s}$ (dashed curve) is nearly constant, which is to be expected since $\tau_{\mathrm{d}}$ is much larger than $\tau_{\text {rec }}$ and $\tau_{\text {ap }}$. Therefore, on average each cell is fully recovered when it is triggered again by another photon.

For $\tau_{\mathrm{d}}=0, \bar{N}_{\text {oct }}=0$, and $\bar{N}_{\text {ap }}=0$ (solid curve), the model predicts the same response as the simple exponential model (dashed-dotted curve), as expected.

For $\tau_{\mathrm{d}}=0$ including afterpulsing and crosstalk (dotted curve), the response converges toward the case that $\tau_{\mathrm{d}}=10 \mu \mathrm{s}$ for small $N_{\mathrm{ph}}$ and toward the case that $\tau_{\mathrm{d}}=0, \bar{N}_{\text {oct }}=0$, and $\bar{N}_{\text {ap }}=0$ for large $N_{\text {ph}}$. In the first case saturation is absent, while in the second case the recovery of the cells is negligible as the light pulse is instantaneous. Therefore, for large $N_{\mathrm{ph}}$ the response converges toward $N_{\text {cells }} / \varepsilon f N_{\text {ph }}$ when afterpulsing and crosstalk are neglected, and toward $\xi_{1} N_{\text {cells }} / \varepsilon f N_{\mathrm{ph}}$ when afterpulsing and crosstalk are included. Consequently, both curves converge toward each other and toward 0 . This is indeed observed in Fig. 8. For completeness, it should be noted that 
TABLE II

SCINTILLATOR Light YIELDS AND DECAY TIMES

\begin{tabular}{llcccccc}
\hline \hline Quantity & Unit & BGO & CsI:Tl & NaI:Tl & LYSO & LaBr$_{3}: C e$ & Reference \\
\hline Light yield, $Y$ & $(\mathrm{ph} / \mathrm{keV})$ & 6.3 & 56 & 53 & 27 & 70 & {$[37]$} \\
Decay time, $\tau_{\mathrm{d}}$ & $(\mathrm{ns})$ & 300 & 1000 & 250 & 41 & 16 & {$[38]$} \\
\hline \hline
\end{tabular}

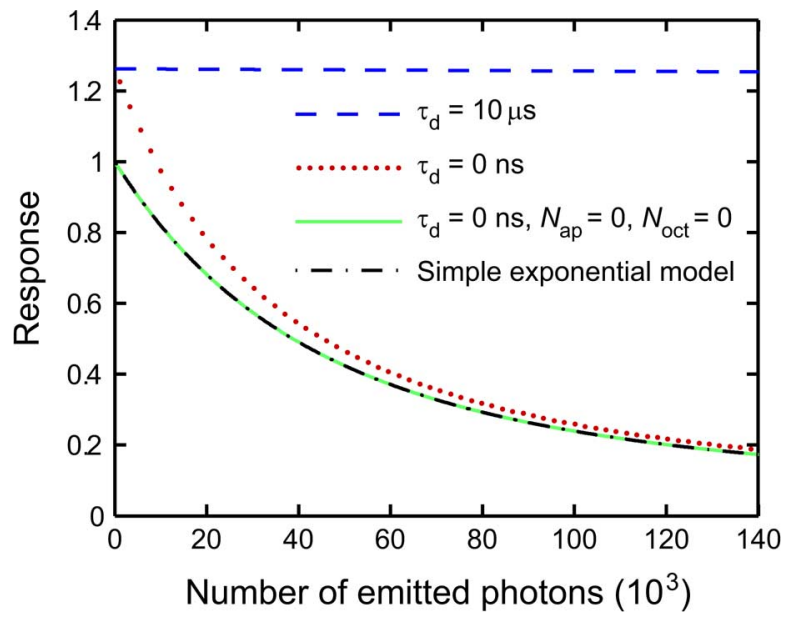

Fig. 8. The modeled SiPM response as a function of the number of emitted photons $N_{\mathrm{ph}}$, for $\tau_{\mathrm{d}}=10 \mu \mathrm{s}$ (dashed curve), for $\tau_{\mathrm{d}}=0$ with (dotted curve), and without (solid curve) afterpulsing and crosstalk, using the SiPM parameters and the detector PDE determined for detector 1 . The response $\chi_{\text {lower }}$ calculated with the simple exponential model (3) is also shown (dashed-dotted curve).

the simplified model for fast light pulses (21) gives the same result as the full model (18) if $\tau_{\mathrm{d}}=0$.

\section{E. Response Predictions for Several Scintillators}

Finally, having compared the model with experiments and several a priori known cases, its application is demonstrated by predicting the response of the Hamamatsu MPPC S10362-33050C SiPM to five common scintillators, viz. BGO, CsI:Tl, NaI:Tl, LSO:Ce, and $\mathrm{LaBr}_{3}$ :Ce, irradiated with $511 \mathrm{keV}$ gamma quanta as used in positron emission tomography (PET). Since the detector PDE, $\varepsilon \cdot f$, depends on the detector geometry, the use of reflecting materials, etc., the response is calculated as a function of $\varepsilon \cdot f$. The values of the light yield $Y$ and the decay time $\tau_{\mathrm{d}}$ of each scintillator, obtained from literature, are listed in Table II. In order for the cumulative distribution function of the number of photon-induced triggers per cell to reach $>99 \%$ for each scintillator, the values of $\xi_{i}$ were fitted and extrapolated up to $i=18$ where necessary. It should be noted that in case of scintillators with longer decay time constants, dark counts and afterpulses with longer time constants may have to be taken into account for more accurate results. The resulting responses are presented in Fig. 9.

It appears that the response for BGO is almost independent of $\varepsilon \cdot f$. Despite the higher light yield of CsI:Tl, its response is also nearly constant due its long decay time. In contrast, a significant reduction of the response may be expected for fast and bright scintillators such as LYSO and $\mathrm{LaBr}_{3}$ :Ce, depending on the detector PDE.

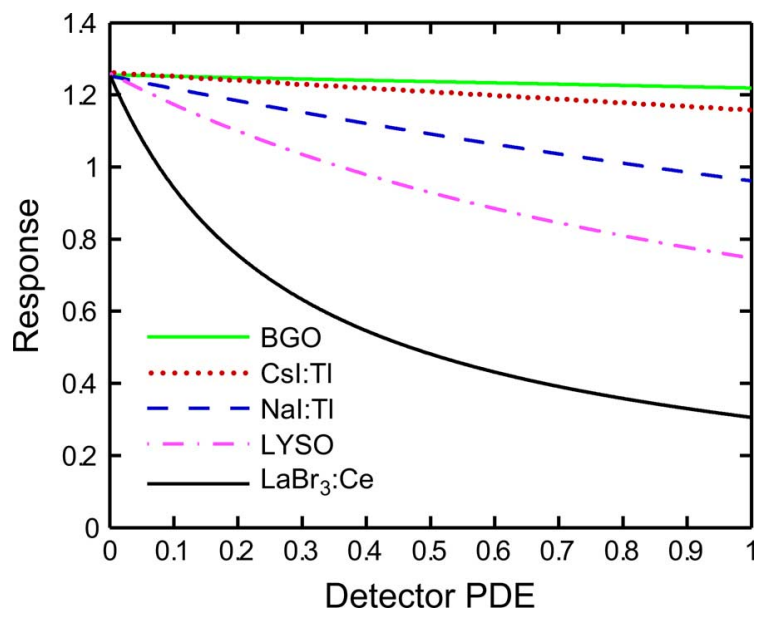

Fig. 9. Predicted response of a Hamamatsu MPPC S10362-33-050C SiPM as a function of the detector PDE, $\varepsilon \cdot f$, for various scintillators irradiated with 511 $\mathrm{keV}$ gamma quanta. The light yield and decay time of each scintillator is given in Table II.

\section{CONCLUSION}

A model of the response of SiPMs that is generally applicable to exponentially decaying light pulses has been developed and validated. In contrast to existing models based on very simple assumptions, it accounts for the total number and the temporal distribution of the incident photons as well as for the SiPM recovery time, afterpulsing, crosstalk, and their cross correlations. This allows for much more accurate calculations on the performance of SiPMs.

The model was shown to agree well with response measurements on two Hamamatsu MPPC S10362-33-050C SiPMs coupled to $\mathrm{LaBr}_{3}: \mathrm{Ce}$ crystals. It is also in agreement with several cases for which the response is known a priori. It was shown how the model can be simplified in the case of very short (e.g., laser) light pulses.

To demonstrate the use of the model, the response of the Hamamatsu MPPC S10362-33-050C SiPM to various scintillators was predicted. Another application of the response model was demonstrated during its validation, where it was used to determine the detector PDE, which otherwise would have been difficult to measure (see Sections IV-C. and V-C).

Further development of the response model, e.g., by including dark counts and the rise time of the current signal through the quench resistor, might make the model even more accurate. However, this would also make the equations in Appendix A more complex.

As a final remark, it is noted that the $\mathrm{C}$ source files, each including a MATLAB gateway routine, for the calculation of 


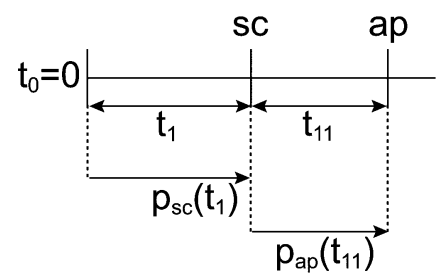

Fig. 10. Time line corresponding to $\psi_{1,1}$ : A scintillation pulse starts at $t_{0}=0$. One of the scintillation photons (sc) causes a trigger after some time interval $t_{1}$, with probability $p_{\mathrm{sc}}\left(t_{1}\right)$. This photon-induced trigger is followed by an afterpulse (ap) after some time interval $t_{11}$, with probability $p_{\text {ap }}\left(t_{11}\right)$.

$\xi_{i}$ for $1 \leq i \leq 9$ according to Section III-C and Appendix A, are available via the corresponding author.

\section{APPENDIX A}

This Appendix shows the derivation of $\psi_{i, j}, \psi_{i, j, k}, \ldots$ for $1 \leq i \leq 4$. Higher order terms can be obtained by logically extending the results of these first four terms.

\section{A. $i=1$}

The expectation value $\psi_{1, j}$ of the output charge of a single cell due to a single photon-induced trigger giving rise to $j$ afterpulses can be obtained using (4), (7), and (8). For example, $\psi_{1,1}$ equals the integral over the product of the probability $p_{\mathrm{sc}}\left(t_{1}\right)$ that a photon-induced trigger is created after a time interval $t_{1}$ following the start of a light pulse at $t_{0}=0$, and the probability $p_{\text {ap }}\left(t_{11}\right)$ that an afterpulse is created after a time interval $t_{11}$ following $t_{1}$, multiplied by the output charge $N_{\mathrm{f}, \mathrm{eq}, 1}\left(t_{11}\right)$ corresponding to this sequence of events, which is schematically represented in Fig. 10

$$
\psi_{1,1}=1 ! 1 ! \int_{0}^{\infty} \int_{0}^{\infty} p_{\mathrm{sc}}\left(t_{1}\right) p_{\mathrm{ap}}\left(t_{11}\right) N_{\mathrm{f}, \mathrm{eq}, 1}\left(t_{11}\right) d t_{1} d t_{11} .
$$

The possible chronological permutations of the photon-induced triggers and the afterpulses are described by the factorial terms in front of the integrals.

Similarly, $\psi_{1,2}$ is given by

$$
\begin{aligned}
\psi_{1,2}= & 1 ! 2 ! \int_{0}^{\infty} \int_{0}^{\infty} \int_{0}^{\infty} p_{\mathrm{sc}}\left(t_{1}\right) p_{\mathrm{ap}}\left(t_{11}\right) p_{\mathrm{ap}}\left(t_{11}+t_{12}\right) \\
& \times N_{\mathrm{f}, \mathrm{eq}, 1}\left(t_{11}, t_{12}\right) d t_{1} d t_{11} d t_{12}
\end{aligned}
$$

where $t_{12}$ is the time interval between the first and the second afterpulse.
By examining higher order terms and evaluating the integrals, it appears that $\psi_{1, j}$ can be written as

$$
\begin{aligned}
\psi_{1, j}=1 ! j !\left(\frac{1}{\tau_{\mathrm{ap}}}\right)^{j} & {\left[\prod_{a_{1}=1}^{j} h\left(a_{1}, 0,0\right)\right.} \\
& \left.\times\left\{1+j-\sum_{a_{1}=1}^{j} \frac{h\left(a_{1}, 0,1\right)}{h\left(a_{1}, 0,0\right)}\right\}\right]
\end{aligned}
$$

where $a_{1}$ is an integer used for summing as well as multiplication, and $h(a, b, c)$ is defined as

$$
h(a, b, c)=\frac{\tau_{\mathrm{d}} \tau_{\mathrm{ap}} \tau_{\mathrm{rec}}}{a \tau_{\mathrm{d}} \tau_{\mathrm{rec}}+b \tau_{\mathrm{ap}} \tau_{\mathrm{rec}}+c \tau_{\mathrm{d}} \tau_{\mathrm{ap}}}
$$

B. $i=2$

The expectation value $\psi_{2, j, k}$ of the output charge of a single cell due to two photon-induced triggers giving rise to $j$ and $k$ afterpulses, respectively, is again calculated by integrating over the probabilities of all possible sequences of events. For example

$$
\begin{aligned}
\psi_{2,1,1}= & 2 ! 1 ! 1 ! \int_{0}^{\infty} \int_{0}^{\infty} \int_{0}^{\infty} \int_{0}^{\infty} q\left(t_{1}, t_{11}, t_{2}, t_{21}\right) \\
& \times N_{\mathrm{f}, \mathrm{eq}, 1}\left(t_{11}, t_{2}, t_{21}\right) d t_{1} d t_{11} d t_{2} d t_{21}
\end{aligned}
$$

where $q\left(t_{1}, t_{11}, t_{2}, t_{21}\right)$ denotes the three possible sequences of events

$$
\begin{aligned}
q\left(t_{1},\right. & \left.t_{11}, t_{2}, t_{21}\right) \\
= & p_{\mathrm{sc}}\left(t_{1}\right) p_{\mathrm{ap}}\left(t_{11}\right) p_{\mathrm{sc}}\left(t_{1}+t_{11}+t_{2}\right) p_{\mathrm{ap}}\left(t_{21}\right) \\
& +p_{\mathrm{sc}}\left(t_{1}\right) p_{\mathrm{sc}}\left(t_{1}+t_{2}\right) p_{\mathrm{ap}}\left(t_{2}+t_{11}\right) p_{\mathrm{ap}}\left(t_{11}+t_{21}\right) \\
& +p_{\mathrm{sc}}\left(t_{1}\right) p_{\mathrm{sc}}\left(t_{1}+t_{2}\right) p_{\mathrm{ap}}\left(t_{21}\right) p_{\mathrm{ap}}\left(t_{2}+t_{21}+t_{11}\right) .
\end{aligned}
$$

The first of these three sequences is schematically represented in Fig. 11.

By examining higher order terms and evaluating the integrals, it appears that $\psi_{2, j, k}$ can be written as (33), shown at the bottom of the page, where $a_{1}, a_{2}$, and $j_{1}$ are integers used for summing as well as multiplication, and $h(a, b, c)$ has been defined in (30).

C. $i=3$

The expectation value $\psi_{3, j, k, l}$ of the output charge of a single cell due to three photon-induced triggers giving rise to $j, k$,

$$
\psi_{2, j, k}=2 ! j ! k !\left(\frac{1}{\tau_{\mathrm{d}}}\right)\left(\frac{1}{\tau_{\mathrm{ap}}}\right)^{j+k} \frac{1}{2} \sum_{j_{1}=0}^{k}\left[\begin{array}{c}
\left.\begin{array}{c}
j+j_{1} \\
j
\end{array}\right) \\
\prod_{a_{1}=1}^{j+j_{1}} h\left(a_{1}, 0,0\right) \prod_{a_{2}=j_{1}}^{k} h\left(a_{2}, 1,0\right) \\
\left.2+j+k-\sum_{a_{1}=1}^{j+j_{1}} \frac{h\left(a_{1}, 0,1\right)}{h\left(a_{1}, 0,0\right)}-\sum_{a_{2}=j_{1}}^{k} \frac{h\left(a_{2}, 1,1\right)}{h\left(a_{2}, 1,0\right)}\right\}
\end{array}\right]
$$




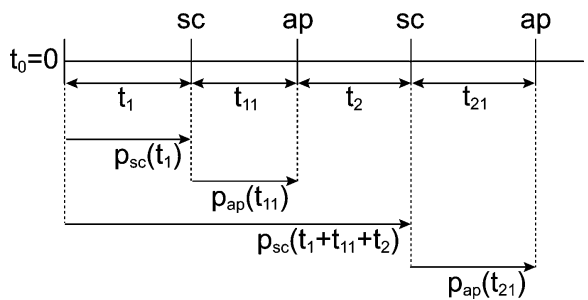

Fig. 11. Timeline of one of the three possible sequences of events corresponding to $\psi_{2,1,1}$. A scintillation pulse starts at $t_{0}=0$. One of the scintillation photons $(\mathrm{sc})$ causes a trigger after some time interval $t_{1}$, with probability $p_{\mathrm{sc}}\left(t_{1}\right)$. This photon-induced trigger is followed by an afterpulse (ap) after some time interval $t_{11}$, with probability $p_{\text {ap }}\left(t_{11}\right)$. The afterpulse is followed by a second absorbed photon causing a trigger after some time interval $t_{2}$, with probability $p_{\mathrm{sc}}\left(t_{1}+t_{11}+t_{2}\right)$. This second photon-induced trigger is followed by an afterpulse after some time interval $t_{21}$, with probability $p_{\text {ap }}\left(t_{21}\right)$.

and $l$ afterpulses, respectively, is obtained similarly. Since the formulas analogous to (31) and (32) for $\psi_{3,1,1,1}$ contain many terms, only the end result is given in (34), shown at the bottom of the page, where $a_{1}, a_{2}, a_{3}, j_{1}$, and $k_{1}$ are integers used for summing and multiplication.

D. $i=4$

The expectation value $\psi_{4, j, k, l, m}$ of the output charge of a single cell due to four photon-induced triggers giving rise to $j, k, l$, and $m$ afterpulses, respectively, is obtained as (35),

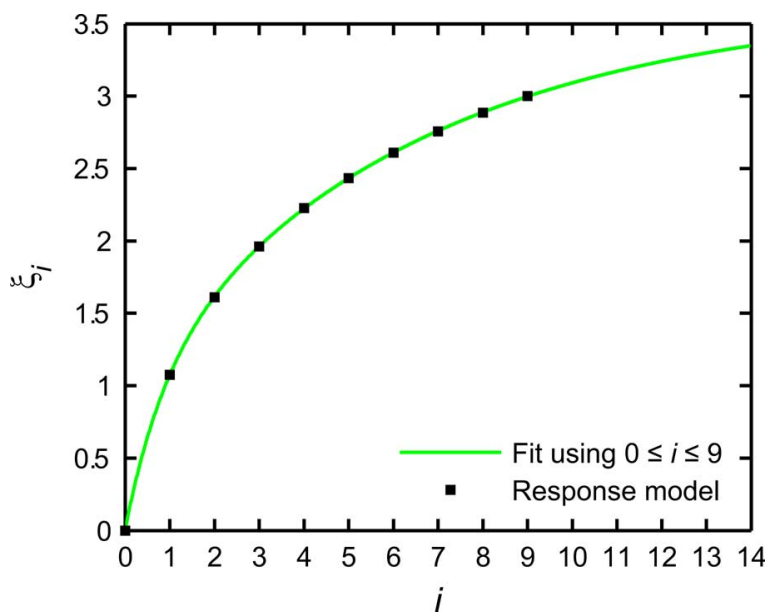

Fig. 12. Modeled values of $\xi_{i}$ as a function of the number of photon-induced triggers in a single cell $i$ (black squares) fitted using $0 \leq i \leq 9$ and extrapolated to $i=14$ (solid curve)

shown at the bottom of the page, where $a_{1}, a_{2}, a_{3}, a_{4}, j_{1}, k_{1}$, and $l_{1}$ are integers used for summing and multiplication.

\section{APPENDIX B}

The computation of $\xi_{i}$ may take a very long time if the number of photon-induced triggers in a single cell $i>9$. As an alternative, additional terms may be obtained by fitting and extrap-

$$
\begin{aligned}
& {\left[\left(\begin{array}{c}
k+k_{1} \\
k
\end{array}\right)\left(\begin{array}{c}
j+j_{1} \\
j
\end{array}\right)\right.} \\
& \times \prod_{a_{1}=1}^{j+j_{1}} h\left(a_{1}, 0,0\right) \prod_{a_{2}=j_{1}}^{k+k_{1}} h\left(a_{2}, 1,0\right) \prod_{a_{3}=k_{1}}^{l} h\left(a_{3}, 2,0\right) \\
& \psi_{3, j, k, l}=3 ! j ! k ! l !\left(\frac{1}{\tau_{d}}\right)^{2}\left(\frac{1}{\tau_{\mathrm{ap}}}\right)^{j+k+l} \frac{1}{3} \times \sum_{k_{1}=0}^{l} \sum_{j_{1}=0}^{k+k_{1}} \\
& \times\left\{\begin{array}{c}
3+j+k+l-\sum_{a_{1}=1}^{j+j_{1}} \frac{h\left(a_{1}, 0,1\right)}{h\left(a_{1}, 0,0\right)} \\
-\sum_{a_{2}=j_{1}}^{k+k_{1}} \frac{h\left(a_{2}, 1,1\right)}{h\left(a_{2}, 1,0\right)}-\sum_{a_{3}=k_{1}}^{l} \frac{h\left(a_{3}, 2,1\right)}{h\left(a_{3}, 2,0\right)}
\end{array}\right\}
\end{aligned}
$$

$$
\begin{aligned}
& \psi_{4, j, k, l, m}=4 ! j ! k ! l ! m !\left(\frac{1}{\tau_{d}}\right)^{3}\left(\frac{1}{\tau_{\text {ap }}}\right)^{j+k+l+m} \frac{1}{4} \\
& \times \sum_{l_{1}=0}^{m} \sum_{k_{1}=0}^{l+l_{1}} \sum_{j_{1}=0}^{k+k_{1}}\left[\begin{array}{c}
\left(\begin{array}{c}
l+l_{1} \\
l
\end{array}\right)\left(\begin{array}{c}
k+k_{1} \\
k
\end{array}\right)\left(\begin{array}{c}
j+j_{1} \\
j
\end{array}\right) \prod_{a_{1}=1}^{j+j_{1}} h\left(a_{1}, 0,0\right) \\
\times \prod_{a_{2}=j_{1}}^{k+k_{1}} h\left(a_{2}, 1,0\right) \prod_{a_{3}=k_{1}}^{l+l_{1}} h\left(a_{3}, 2,0\right) \prod_{a_{4}=l_{1}}^{m} h\left(a_{4}, 3,0\right) \\
\times\left\{\begin{array}{c}
4+j+k+l+m \\
-\sum_{a_{1}=1}^{j+j_{1}} \frac{h\left(a_{1}, 0,1\right)}{h\left(a_{1}, 0,0\right)}-\sum_{a_{2}=j_{1}}^{k+k_{1}} \frac{h\left(a_{2}, 1,1\right)}{h\left(a_{2}, 1,0\right)} \\
-\sum_{a_{3}=k_{1}}^{l+l_{1}} \frac{h\left(a_{3}, 2,1\right)}{h\left(a_{3}, 2,0\right)}-\sum_{a_{4}=l_{1}}^{m} \frac{h\left(a_{4}, 3,1\right)}{h\left(a_{4}, 3,0\right)}
\end{array}\right\}
\end{array}\right]
\end{aligned}
$$


olating $\xi_{i}$ as a function of $i$. This is illustrated for the case of detector 1 , for which the parameters have been determined in Section V. The following function fits the modeled values of $\xi_{i}$ well for this detector:

$$
\xi_{\text {fit }}\left(i, h_{1}, h_{2}, h_{3}\right)=\left(1-e^{-\frac{i}{h_{1}}}\right)+h_{2}\left(1-e^{-\frac{i}{h_{3}}}\right)
$$

where $h_{1}, h_{2}$, and $h_{3}$ are the fit parameters. It should be noted that this function has no physical meaning.

Fig. 12 shows the fit (solid curve) and the modeled values of $\xi_{i}$ (black squares). Since the curve becomes less steep for larger values of $i$, it is expected that possible deviations of the extrapolated values of $\xi_{i}$ from the modeled ones become smaller with increasing $i$.

\section{REFERENCES}

[1] P. P. Antich, E. N. Tsyganov, N. A. Malakhov, and Z. Y. Sadygov, "Avalanche photo diode with local negative feedback sensitive to UV, blue and green light," Nucl. Instrum. Methods Phys. Res. A, vol. 389, pp. 491-498, 1997.

[2] G. Bondarenko et al., "Limited geiger-mode microcell silicon photodiode: New results," Nucl. Instrum. Methods Phys. Res. A, vol. 442, pp. 187-192, 2000.

[3] V. Golovin and V. Saveliev, "Novel type of avalanche photodetector with Geiger mode operation," Nucl. Instrum. Methods Phys. Res. A, vol. 518, pp. 560-564, 2004.

[4] K. J. Hong et al., "Development of PET using $4 \times 4$ array of large size geiger-mode avalanche photodiode," in IEEE Nucl. Sci. Symp. Conf. Record 2009, Orlando, FL, pp. 3032-3037, (M09-8).

[5] M. Mazzillo et al., "Silicon photomultiplier technology at STMicroelectronics," IEEE Trans. Nucl. Sci., vol. 56, no. 4, pp. 2434-2442, Aug. 2009.

[6] C. Piemonte et al., "Recent developments on silicon photomultipliers produced at FBK-irst," in IEEE Nucl. Sci. Symp. Conf. Record 2007, Honolulu, HI, pp. 2089-2092, (N41-2).

[7] K. Yamamoto et al., "Development of multi-pixel photon counter (MPPC)," in Nucl. Sci. Symp. Conf. Record 2007, Honolulu, HI, pp. 1511-1515, (N24-292).

[8] P. Buzhan et al., "Silicon photomultiplier and its possible applications," Nucl. Instrum. Methods Phys. Res. A, vol. 504, pp. 48-52, 2003.

[9] B. Dolgoshein et al., "Status report on silicon photomultiplier development and its applications," Nucl. Instrum. Methods Phys. Res. A, vol. 563, pp. 368-376, 2006.

[10] D. Renker, "New developments on photosensors for particle physics," Nucl. Instrum. Methods Phys. Res. A, vol. 598, pp. 207-212, 2009.

[11] P. Achenbach, A. S. Lorente, S. S. Majos, and J. Pochodzalla, "Future use of silicon photomultipliers for KAOS at MAMI and PANDA at FAIR," Nucl. Instrum. Methods Phys. Res. A, vol. 610, pp. 358-361, 2009.

[12] V. Andreev et al., "A high-granularity scintillator calorimeter readout with silicon photomultipliers," Nucl. Instrum. Methods Phys. Res. A, vol. 540, pp. 368-380, 2005.

[13] M. Danilov, CALICE Collaboration, "Scintillator tile hadron calorimeter with novel SiPM readout," Nucl. Instrum. Methods Phys. Res. A, vol. 581, pp. 451-456, 2007,

[14] S. Korpar et al., "Measurement of cherenkov photons with silicon photomultipliers," Nucl. Instrum. Methods Phys. Res. A, vol. 594, pp. $13-17,2008$.

[15] S. Schuwalow, "Calorimetry at the ILC detectors," Nucl. Instrum. Methods Phys. Res. A, vol. 598, pp. 258-263, 2009.

[16] A. N. Otte et al., "A test of silicon photomultipliers as readout for PET," Nucl. Instrum. Methods Phys. Res. A, vol. 545, pp. 705-715, 2005.

[17] D. R. Schaart et al., "A novel, SiPM-array-based, monolithic scintillator detector for PET," Phys. Med. Biol., vol. 54, pp. 3501-3512, 2009.
[18] A. N. Otte et al., "Focal plane detectors for the advanced gamma-ray imaging system (AGIS)," in Proc. 4th Int. Meeting High Energy Gamma-Ray Astronomy, 2009, CP1085.

[19] A. N. Otte et al., "Prospects of using silicon photomultipliers for the astroparticle physics experiments EUSO and MAGIC," IEEE Trans. Nucl. Sci., vol. 53, no. 2, pp. 636-640, Apr. 2006.

[20] I. Afek, A. Natan, O. Ambar, and Y. Silberberg, "Quantum state measurements using multipixel photon detectors," Phys. Rev. A, vol. 79, p. 043830, 2009.

[21] S. Arnon and D. Kedar, "Non-line-of-sight underwater optical wireless communication network," J. Opt. Soc. Amer. A, vol. 26, no. 3, pp. 530-539, Mar. 2009.

[22] K. Tak Son and C. C. Lee, "Multiple-target laser range-finding receivers using silicon photomultiplier arrays," in Proc. Electron. Compon. Technol. Conf., 2009, pp. 2131-2136.

[23] P. Eraerds, M. Legré, A. Rochas, H. Zbinden, and N. Gisin, "SiPM for fast photon-counting and multiphoton detection," Opt. Express, vol. 15, no. 22, pp. 14539-14549, Oct. 2007.

[24] M. Song, E. Won, and T. H. Yoon, "Large dynamic range photon detector with a temperature-stabilized Si-based multi-pixel photon counter," Opt. Express, vol. 15, no. 25, pp. 17099-17105, Dec. 2007.

[25] A. Stoykov, Y. Musienko, A. Kuznetsov, S. Reucroft, and J. Swain, "On the limited amplitude resolution of multipixel geiger-mode APDs," $J$. Instrum., vol. 2, p. P06005, Jun. 2007.

[26] J. Barral, "Study of silicon photomultipliers," Ph.D. dissertation, Ecole Polytechnique, Paris, France, 2004.

[27] V. C. Spanoudaki, "Development and performance studies of a small animal positron emission tomograph with individual crystal readout and depth of interaction information and studies of novel detector technologies in medical imaging," Ph.D. dissertation, Technische Universität München, München, Germany, 2008.

[28] F. Corsi et al., "Modelling a silicon photomultiplier (SiPM) as a signal source for optimum front-end design," Nucl. Instrum. Methods Phys. Res. A, vol. 572, pp. 416-418, 2007.

[29] S. Seifert et al., "Simulation of silicon photomultiplier signals," IEEE Trans. Nucl. Sci., vol. 56, no. 6, pp. 3726-3733, Dec. 2009.

[30] S. Cova, M. Ghioni, A. Lacaita, C. Samori, and F. Zappa, "Avalanche photodiodes and quenching circuits for single-photon detection," Appl. Opt., vol. 35, no. 12, pp. 1956-1976, Apr. 1996.

[31] R. H. Haitz, "Model for the electrical behavior of a microplasma," $J$. Appl. Phys., vol. 35, no. 5, pp. 1370-1376, May 1964.

[32] Y. Du and F. Retière, "After-pulsing and cross-talk in multi-pixel photon counters," Nucl. Instrum. Methods Phys. Res. A, vol. 596, pp. 396-401, 2008.

[33] S. Cova, A. Lacaita, and G. Ripamonti, "Trapping phenomena in avalanche photodiodes on nanosecond scale," IEEE Electron Device Lett., vol. 12, no. 12, pp. 685-687, Dec. 1991.

[34] A. L. Lacaita, F. Zappa, S. Bigliardi, and M. Manfredi, "On the bremsstrahlung origin of hot-carrier-induced photons in silicon devices," IEEE Trans. Electron Devices, vol. 40, no. 3, pp. 577-582, Mar. 1993.

[35] I. Rech et al., "Optical crosstalk in single photon avalanche diode arrays: A new complete model," Opt. Express, vol. 16, no. 12, pp. 8381-8394, Jun. 2008

[36] "Hamamatsu MPPC S10362-33 series S103931 series product sheets, Cat. No. KAPD1023E04" Hamamatsu Photonics K.K., Solid State Division, Aug. 2009.

[37] J. T. M. de Haas and P. Dorenbos, "Advances in yield calibration of scintillators," IEEE Trans. Nucl. Sci., vol. 55, no. 3, pp. 1086-1092, Jun. 2008.

[38] "Saint-Gobain Material Product Sheets for NaI:Tl (10-05), BGO (0307), CsI:Tl (05-07), LYSO (07-07), and $\mathrm{LaBr}_{3}: \mathrm{Ce}$ (12-07)" [Online]. Available: http://www.detectors.saint-gobain.com

[39] A. Owens et al., "The hard X-ray response of Ce-doped lanthanum halide scintillators," Nucl. Instrum. Methods Phys. Res. A, vol. 574, pp. 158-162, 2007.

[40] G. Bizarri, J. T. M. de Haas, P. Dorenbos, and C. W. E. van Eijk, "Scintillation properties of $\oslash 1 \times 1 \mathrm{Inch}^{3} \mathrm{LaBr}_{3}: 5 \% \mathrm{Ce}^{3+}$ crystal," IEEE Trans. Nucl. Sci., vol. 53, no. 2, pp. 615-619, Apr. 2006.

[41] "Hamamatsu MPPC product sheets, Cat. No. KAPD0002E03," Hamamatsu Photonics K.K., Solid State Division, Jan. 2008. 